\title{
Performances de croissance des ovins Djallonké en milieu villageois dans le département du pool (Congo Brazzaville)
}

\author{
Richard MABEKI MISSOKO1, Paul MOPOUNDZA², Dora Chérita EKOU³, Sylvie Nadège MASSAMBA4 \\ et Parisse AKOUANGO5 \\ 1 Assistant au Laboratoire des productions animales et biodiversité, École Nationale Supérieure d'Agronomie et de \\ Foresterie Université Marien Ngouabi. BP. 69. \\ 2 Maitre Assistant au Laboratoire des productions animales et biodiversité, École Nationale Supérieure d'Agronomie \\ et de Foresterie Université Marien Ngouabi. BP. 69. \\ 3 Assistant au Laboratoire des productions animales et biodiversité, École Nationale Supérieure d'Agronomie et de \\ Foresterie Université Marien Ngouabi. BP. 69. \\ 4 Étudiante en Master 2 à l'École Nationale Supérieure d'Agronomie et de Foresterie Université Marien Ngouabi. BP. \\ 69. \\ 5 Professeur des universités en zootechnie et biodiversité, École Nationale Supérieure d'Agronomie et de Foresterie, \\ Université Marien Ngouabi. BP. 69 .Tél : (00242)066698519 ; parakouango@yahoo.fr \\ Auteur Correspondant : Richard MISSOKO MABEKI ; Email : richard_mabeki@yahoo.fr; Tél : (00242) 068559504
}

Original submitted in on $6^{\text {th }}$ August 2018. Published online at www.m.elewa.org on $30^{\text {th }}$ November 2018 https://dx.doi.org/10.4314/jab.v131i1.4

\section{RÉSUMÉ}

Objectif: Cette étude a eu pour but d'évaluer les performances de croissance des agneaux de race Djallonké dans le département du pool district d'Ignié en milieu paysan.

Méthodologie et résultats: Elle a porté sur 74 agneaux issus de 68 brebis et 7 males dans la période d'Août 2017 à Mars 2018. Les données zootechniques (poids vif, gain moyen quotidien) ont été recueillies hebdomadairement de la naissance à 180 jours d'âge. Les résultats obtenus ont montré une différence significative de poids à la naissance. Les agneaux issus des naissances simples étaient plus lourds $(1,95 \pm 0,13 \mathrm{~kg})$ que les agneaux des naissances doubles et triples $(1,05 \pm 0,14 \mathrm{~kg}$ et $1 \pm 00)$. A partir de 120 jours, les poids des agneaux issus des triples naissances avaient augmenté significativement $(12,5 \pm 0,82 \mathrm{~kg})$. Le facteur sexe a influencé de manière significative la croissance pondérale des agneaux de la naissance à J180. Le GMQ (Gain Moyen Quotidien) a été influencé par le type de naissance. Les agneaux nés uniques ont montré des $G M Q$ supérieurs suivi des naissances doubles et triples. L'expérience des mères a montré des GMQ plus important chez les agneaux issus des mères dont l'âge est compris entre 3 et 4ans alors que les plus faibles ont été enregistrés chez les agneaux issus des mères expérimentées (5ans). Le facteur sexe n'a pas influencé le gain moyen quotidien à 30 jours $(p>0,05)$. II a été cependant déterminant par la suite (30-180j) auquel le GMQ des mâles a fortement dominé sur celui des femelles.

Conclusion et application des résultats: Les résultats obtenus dans cette étude montrent une bonne disposition de la race Djallonké en milieu tropical. II sied néanmoins de noter que, les performances (croissance pondérale et le gain moyen quotidien) ont été plus meilleures chez les portées uniques. La 

dans le département du pool (Congo Brazzaville).

croissance pondérale et le gain moyen quotidien élevés chez les naissances triples met en éclair le problème de la compétition au niveau de leur alimentation avant sevrage au sein d'une même portée.

Mots clés : performances-ovins Djallonké-milieu villageois-département du pool

\section{ABSTRACT}

Objective: The purpose of this study was to evaluate the performances of growth of the lambs of Djallonké race in the department of the pool district of Ignié in country medium.

Methodology and results: It related to 74 lambs resulting from 68 ewes and 7 rams during the time from August 2017 to March 2018. The zootechnical data (live weight, daily average profit) were collected weekly of the birth at 180 days of age. The results showed a significant difference in weight to the birth. The lambs resulting from the simple births were heavier $(1,95 \pm 0,13 \mathrm{~kg})$ that lambs of the births double and triple $(1,05 \pm 0,14 \mathrm{~kg}$ and $1 \pm 00)$. From 120 days, the weights of the lambs resulting from the triple births had increased significantly $(12,5 \pm 0,82 \mathrm{~kg})$. The factor sex significantly influenced the ponderal growth of the lambs of the birth with $\mathrm{J} 180$. The GMQ was influenced by the type of birth. The lambs born single showed followed higher GMQ by the double and triple births. The experiment of the mothers showed more important GMQ in the lambs resulting from the mothers whose age lies between 3 and 4ans whereas weakest were recorded in the lambs resulting from the experienced mothers (5ans). The factor sex did not influence the daily average profit at 30 days $(p>0,05)$. It was however determining thereafter $(30-180 j)$ to which the GMQ of the males strongly dominated over that of the females.

Conclusion and application of results: The results got in this study show a good provision of the Djallonké race in tropical medium. It sied nevertheless to note that, the performances (ponderal growth and daily average profit) were better at the single ranges. The ponderal growth and the average profit daily high at the triple births put in flash the problem of the competition at the level of their food before weaning within the same range

Keywords: performance-sheep villager-department Djallonké-medium of the pool.

\section{INTRODUCTION}

L'élevage assure une sécurisation des familles et un outil de lutte contre la pauvreté. II est une capitalisation qui permet une diversification des activités et représente un facteur d'intégration économique et sociale (Faye et Alary, 2001). Au Congo cependant, l'élevage occupe une place de faible importance dans l'échelle des activités rurales, comme c'est le cas dans les systèmes agricoles dans lesquels moins de $10 \%$ du revenu brut des ménages provient de l'élevage (Wilson, 1989). En effet, le paysan congolais est avant tout un cultivateur qui vit d'agriculture, de chasse, de cueillette et de pêche. L'élevage des petits ruminants est une activité presque exclusivement masculine au Congo (Amoundala-Matala, 1982; Savou, 1982; Kinga, 1983 ; Batalou-Mbetani et Bourzat, 1989; Mfoukou-Ntsakala, 2010), contrairement à ce qui a été observé en Afrique de l'Ouest où cette activité est tenue par les femmes (Lhoste et al., 1993). Cet élevage qui en milieu traditionnel, est resté pendant longtemps un élevage de prestige a été considéré comme une sorte de capital intervenant à l'occasion de certaines cérémonies comme le mariage, les décès, les fêtes, les réceptions d'un hôte de marque. Les performances de croissance des ovins Djallonké varient en fonction du système d'élevage. Celui-ci peut être pastoral, agropastoral ou amélioré (Wilson, 1983 ; 1989 ; Lebbie et Ramsay, 1999). En raison de leur adaptation aux différentes zones agro-écologiques (Centre international pour l'Élevage en Afrique, 1979 ; Mawuena, 1986 ; 1987 ; Bengaly et al., 1993), les ovins Djallonké sont particulièrement intéressants pour contribuer à atteindre l'objectif fondamental de sécurité alimentaire des populations en protéines animales (Faye et Lhoste, 1999 ; Faye et Alary, 2001) et ainsi sortir de la paupérisation (Ashley et al., 1999). En milieu traditionnel, les facteurs non génétiques (santé, alimentation et 
perturbations climatiques) contribuent à réduire la plupart des caractères de croissance, et doivent être contrôlés (Clément et al., 1997 ; Yapi-Gnaoré, 1997b). Le but de cette étude est de contribuer à

\section{MATÉRIEL ET MÉTHODES}

Zone d'étude : Le Pool est l'un des départements de la République du Congo situé dans la partie sud-ouest du pays. Sa superficie est de 3.395 .500 hectares et une population de 236.595 habitants8 soit une densité de 7 $\mathrm{h} / \mathrm{km}$, ce qui représente environ $10 \%$ de l'espace national congolais. II est situé au sud-ouest du pays, le département du Pool est limité par :

- le département du Plateaux au nord ;

- les contreforts du massif du Chaillu et le département de la Lékoumou au nord-ouest ;

- les plateaux des cataractes et la République Démocratique du Congo au sud ;

- le fleuve-Congo à l'est ;

- le département de la Bouenza à l'ouest.

Le département du Pool est arrosé par deux grands fleuves possédant de nombreux affluents

- le fleuve-Congo : alimenté par des grands cours d'eau comme la Léfini, la Djiri, le Djoué, la Loufoulakari, Louenga, Madzia, Loualou, Djouari, et la Loukouangou. - le fleuve Ndouo-Niari à partir de Mindouli.

Le département du Pool est soumis à un climat du type bas-congolais ou encore soudano-guinéen caractérisé par :

* une longue saison sèche allant de 4 à 5 mois, de Mai à Septembre

* une longue saison des pluies, marquée par une diminution de pluviosité en Janvier et Février, période appelée " petite saison sèche » (ESA, 2012)

La pluviosité annuelle est assez constante dans toute la région. Elle oscille entre 1270 et $1350 \mathrm{~mm}$.

Troupeau : Le troupeau ovin de la ferme Agropastorale de Djomossi a un effectif total de 110 têtes de race

\section{RÉSULTATS}

Croissance pondérale des agneaux de race Djallonké: Le tableau 1 montre l'évolution de la croissance pondérale en fonction du type de naissance des agneaux de la race Djallonké de la naissance à 180 jours. De ce tableau, il ressort que les agneaux nés simple ont présenté les poids significativement différent la compréhension des évolutions des performances pondérales des ovins Djallonké, afin d'aider à l'élaboration de stratégies de valorisation de cette population ovine au Congo Brazzaville.

Djallonké. II est composé de la manière suivante : 7 béliers, 73 brebis, 13 males jeunes, 11 agnèles et 6 agneaux. Pour la présente étude, le suivi des performances de croissance n'a concerné que les agneaux issus des 73 brebis à compter de la date du début de notre étude. Un certain nombre de critères étaient déterminants dans la sélection du troupeau expérimental notamment: Le bon état corporel ou la bonne conformation, la bonne santé et l'intégrité de l'appareil génital.

Conduite de l'alimentation : Les sites sont loin de toutes sortes de l'urbanisation et de l'industrialisation. Le troupeau de notre étude était entretenu sur un mode extensif. L'apport en supplément alimentaire était très rarement assuré. Ainsi, tous les animaux de l'expérimentation profitent exclusivement de pâturages offerts par des parcours naturels de la zone Igné du département du pool.

Variables étudiées: Les agneaux sont identifiés à laide de boucles auriculaires et pesés à la naissance puis à $30 \mathrm{j}$, à $90 \mathrm{j}$, à $120 \mathrm{j}$, à $150 \mathrm{j}$ et $180 \mathrm{j}$. Les gains moyens quotidiens (GMQ) sont calculés à partir des poids aux âges-types correspondants.

Analyses statistiques des données: L'analyse des résultats obtenus et la comparaison des moyennes ont été effectuées par le test d'analyse de variance (ANOVA) au seuil de signification de 0,05 à l'aide du logiciel Statistical Package for the Social Science (SPSS) version 10.0.5 et complété par le test de Duncan lorsque le test d'ANOVA a montré une différence significative.

$(p<0,05)$ à la naissance par rapport aux agneaux nés double et triple. II a été noté cependant un effet contraire à 120jours ou le poids des agneaux nés triple a été significativement différent des naissances simple et double. Un effet non significatif a été noté à J30, J60, $\mathrm{J} 90, \mathrm{~J} 150$ et $\mathrm{J} 180$. 

dans le département du pool (Congo Brazzaville).

Tableau 1 : Évolution de la croissance pondérale en fonction du type de naissance

\begin{tabular}{|l|l|l|l|l|l|}
\hline \multirow{2}{*}{ Paramètre } & \multirow{2}{*}{ Age en mois } & \multicolumn{2}{|l|}{ Mode de naissance } & \multirow{2}{*}{ Signification } \\
\cline { 2 - 6 } & & Unique & Double & Triple & ** \\
\hline \multirow{5}{*}{ Poids en fonction du temps } & $\mathrm{J} 0$ & $1,95 \pm 0,13 \mathrm{~b}$ & $1,05 \pm 0,14 \mathrm{a}$ & $1 \pm 00 \mathrm{a}$ & $\mathrm{N}$ \\
\cline { 2 - 6 } & $\mathrm{J} 30$ & $4,65 \pm 1,78$ & $3,04 \pm 0,76$ & $3,41 \pm 0,80$ & NS \\
\cline { 2 - 6 } & $\mathrm{J} 60$ & $4,67 \pm 1,54$ & $4,75 \pm 1,57$ & $6,71 \pm 1,59$ & NS \\
\cline { 2 - 6 } & $\mathrm{J} 90$ & $7,73 \pm 1,65$ & $6,91 \pm 2,63$ & $9,5 \pm 0,88$ & NS \\
\cline { 2 - 6 } & $\mathrm{J} 120$ & $11,19 \pm 1,64 \mathrm{ab}$ & $9,12 \pm 2,19 \mathrm{a}$ & $12,5 \pm 0,82 \mathrm{~b}$ & ** \\
\cline { 2 - 6 } & $\mathrm{J} 150$ & $13,84 \pm 1,62$ & $12,66 \pm 3,02$ & $14,58 \pm 1,01$ & NS \\
\cline { 2 - 6 } & $\mathrm{J} 180$ & $16,04 \pm 1,26$ & $14,40 \pm 2,66$ & $14,76 \pm 0,86$ & NS \\
\hline
\end{tabular}

$a, b, c$ : Les moyennes suivies de lettres différentes au sein d'une même ligne sont significativement différentes au seuil de $5 \%$

La croissance pondérale des agneaux en fonction de l'expérience des mères est consignée dans le tableau 2. Le poids des agneaux nés des mères dont l'âge tourne autour de 2 à 4 ans a été significativement différent à la naissance $(p<0,05)$ à ceux des agneaux dont les mères ont 1 an et 5 ans. Le non significativité de la croissance pondérale en fonction de l'expérience des mères a été cependant noté de $\mathrm{J} 30$ à $\mathrm{J} 180$.

Tableau 2 : Évolution de la croissance pondérale en fonction de l'âge des brebis

\begin{tabular}{|c|c|c|c|c|c|c|c|}
\hline \multirow{2}{*}{ Paramètre } & \multirow{2}{*}{$\begin{array}{l}\text { Age en } \\
\text { mois }\end{array}$} & \multicolumn{5}{|c|}{ Age de la mère } & \multirow[b]{2}{*}{ Signification } \\
\hline & & 1an & 2ans & 3ans & 4ans & 5ans & \\
\hline \multirow{7}{*}{$\begin{array}{l}\text { Poids en fonction } \\
\text { du temps }\end{array}$} & JO & $1,00 \pm 0,00 a$ & $2,00 \pm 0,00 b$ & $1,5 \pm 0,49 a b$ & $1,68 \pm 0,46 b$ & $1,00 \pm 0,00 a$ & ** \\
\hline & $\mathrm{J} 30$ & $4,5 \pm 0,71$ & $3,46 \pm 0,29$ & $3,65 \pm 1,61$ & $5,14 \pm 1,69$ & $3,42 \pm 0,80$ & NS \\
\hline & $\mathrm{J} 60$ & $5,00 \pm 1,41$ & $4,88 \pm 1,24$ & $4,49 \pm 1,68$ & $5,20 \pm 1,26$ & $6,71 \pm 1,59$ & NS \\
\hline & $\mathrm{J} 90$ & $7,75 \pm 0,35$ & $8,04 \pm 0,41$ & $7,01 \pm 2,30$ & $8,15 \pm 1,73$ & $9,50 \pm 0,88$ & NS \\
\hline & J120 & $10,50 \pm 0,71$ & $10,75 \pm 0,35$ & $9,98 \pm 2,43$ & $11,20 \pm 1,31$ & $12,5 \pm 0,82$ & NS \\
\hline & $\mathrm{J} 150$ & $14,50 \pm 0,71$ & $13,31 \pm 0,97$ & $13,40 \pm 2,77$ & $13,31 \pm 1,29$ & $14,58 \pm 1,01$ & NS \\
\hline & J180 & $17,00 \pm 1,41$ & $16,08 \pm 2,00$ & $15,48 \pm 2,44$ & $14,86 \pm 0,77$ & $14,77 \pm 0,86$ & NS \\
\hline
\end{tabular}

$a, b, c$ : Les moyennes suivies de lettres différentes au sein d'une même ligne sont significativement différentes au seuil de $5 \%$

Bien que le facteur sexe ne soit pas appréhendé de manière explicite dans les études de productivité pondérale, il en a été tenu compte dans ce travail eu égard à son effet mis en évidence sur les poids aux âges types et les gains moyens quotidiens individuels des agneaux (Ben, 1985 ; Aloulou, 1990 et Jmal, 1995). A cet effet, la figure 1 montre l'évolution de la croissance pondérale des agneaux males et femelles de 0 à 180 jours. De cette figure, il ressort que le poids des agneaux males a été largement supérieur à celui des femelles de la naissance jusqu'à l'âge de 6mois. II est cependant à noter qu'à J60, le poids dans les deux sexes se confond $(p>0,05)$ avec une légère dominance du poids des femelles. 


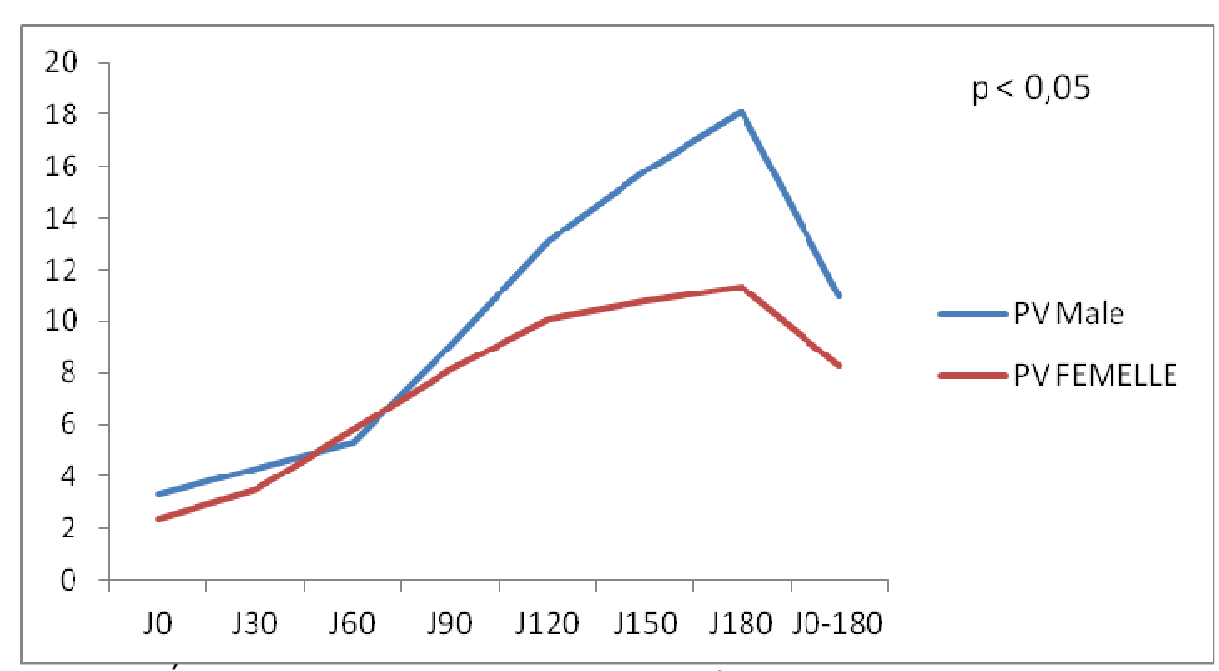

Figure 1 : Évolution de la croissance pondérale en fonction du sexe des agneaux de race Djallonké

Gain moyen quotidien des agneaux de race Djallonké : Les gains moyens quotidiens des agneaux de race Djallonké sont présentés dans le tableau 3 cidessous. II ressort de ce tableau que les GMQ entre 030j et 90-120j ont été significativement différent au plan statistique respectivement pour les agneaux nés simple et double. Cependant, une différence non significative des gains moyens quotidiens des agneaux de race Djallonké est notée entre $30-60 \mathrm{j} ;$ 60-90j ; 120-150j et 150-180j.

Tableau 3 : Évolution du gain moyen quotidien en fonction du type de naissance

\begin{tabular}{|c|c|c|c|c|c|}
\hline \multirow{2}{*}{ Paramètre } & \multirow{2}{*}{ Age en mois } & \multicolumn{3}{|c|}{ Mode de naissance } & \multirow{2}{*}{ Signification } \\
\cline { 3 - 6 } & & Unique & Double & Triple & ${ }^{* *}$ \\
\hline \multirow{4}{*}{ GMQ } & GMQ0-30j & $126,37 \pm 51,96 \mathrm{a}$ & $90,03 \pm 28,54 \mathrm{ab}$ & $41,67 \pm 27,28 \mathrm{~b}$ & $\mathrm{NS}$ \\
\cline { 2 - 6 } & GMQ30-60j & $135,98 \pm 102,20$ & $60,27 \pm 58,79$ & $57,54 \pm 83,40$ & $\mathrm{NS}$ \\
\cline { 2 - 6 } & GMQ60-90j & $137,36 \pm 102,16$ & $60,27 \pm 58,79$ & $27,78 \pm 69,75$ & ${ }^{*}$ \\
\cline { 2 - 6 } & GMQ90-120j & $151,10 \pm 104,03 \mathrm{~b}$ & $87,05 \pm 36,27 \mathrm{ab}$ & $, 81 \pm 82,48 \mathrm{a}$ & $\mathrm{NS}$ \\
\cline { 2 - 6 } & GMQ120-150j & $142,86 \pm 112,94$ & $142,86 \pm 76,36$ & $35,71 \pm 94,49$ & $\mathrm{NS}$ \\
\cline { 2 - 6 } & GMQ150-180j & $152,75 \pm 105,52$ & $153,57 \pm 66,90$ & $119,04 \pm 82,48$ & \\
\hline
\end{tabular}

$a, b, c$ : Les moyennes suivies de lettres différentes au sein d'une même ligne sont significativement différentes au seuil de $5 \%$

Le tableau 4 présente l'évolution du gain moyen quotidien en fonction de l'expérience des brebis. II en résulte de ce tableau que les gains moyens quotidiens des agneaux issus des brebis de 4 et 3 ans ont les
GMQ significativement différent des autres entre 0-30j. Une différence non significative entre 30-60j et la fin de l'expérience a été noté au niveau de ces différents âges des mères.

Tableau 4 : Évolution du gain moyen quotidien en fonction de l'âge des brebis

\begin{tabular}{|c|c|c|c|c|c|c|}
\hline \multirow{2}{*}{ Paramètre } & \multirow{2}{*}{ Age en mois } & \multicolumn{4}{|c|}{ Age de la mère } & \multirow[b]{2}{*}{ Signification } \\
\hline & & 2ans & 3ans & 4ans & 5ans & \\
\hline \multirow{6}{*}{ GMQ } & GMQ0-30j & $91,27 \pm 18,18 a b$ & $113,09 \pm 56,57 b$ & $123,81 \pm 29,88 b$ & $41,67 \pm 27,28 a$ & ** \\
\hline & GMQ30-60j & $95,24 \pm 41,24$ & $104,39 \pm 115,00$ & $121,43 \pm 59,76$ & $57,54 \pm 83,40$ & NS \\
\hline & GMQ60-90j & $101,19 \pm 37,17$ & $98,90 \pm 112,85$ & $132,14 \pm 55,90$ & $27,78 \pm 69,75$ & NS \\
\hline & GMQ90-120j & $101,19 \pm 37,17$ & $135,99 \pm 107,28$ & $117,86 \pm 61,34$ & $23,81 \pm 82,48$ & NS \\
\hline & GMQ120-150j & $95,24 \pm 41,24$ & $137,36 \pm 110,89$ & $185,71 \pm 81,44$ & $35,71 \pm 94,49$ & NS \\
\hline & GMQ150-180j & $90,48 \pm 45,92$ & $154,94 \pm 98,78$ & $185,7123 \pm 81,44$ & $119,04 \pm 82,48$ & NS \\
\hline
\end{tabular}

a, b, c : Les moyennes suivies de lettres différentes au sein d'une même ligne sont significativement différentes au seuil de 5 \% 
La figure 2 montre l'évolution du gain moyen quotidien des agneaux de race Djallonké en fonction du sexe. De cette figure, il en résulte que les agneaux males ont enregistré un GMQ significativement élevé $(P<0,05)$ que celui des femelles de J30 à J180. A la naissance, les gains moyens quotidiens des agneaux ont augmenté de façon similaire dans les deux sexes $(P>$ $0,05)$ bien que cette évolution ait semblé plus importante chez les males.

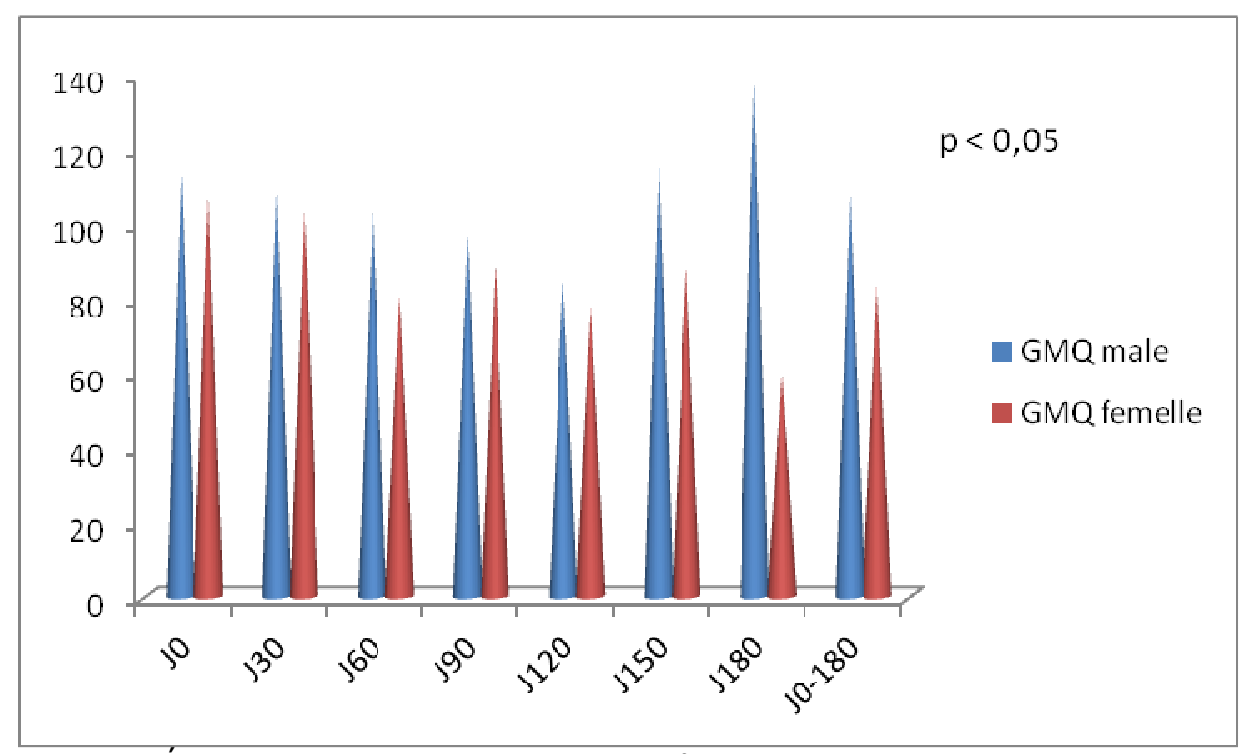

Figure 2 : Évolution du gain moyen quotidien en fonction du sexe des agneaux

\section{DISCUSSION}

Le type de naissance a influencé significativement les poids vifs moyens des agneaux à la naissance et à $\mathrm{J} 120(\mathrm{p}<0,05)$. À la naissance, les poids ont été respectivement de 1,$95 ; 1,05$ et $1 \mathrm{~kg}$ pour les agneaux nés simples, doubles et triples et à J120 de 11,$19 ; 9,12$ et $12,5 \mathrm{~kg}$ respectivement pour les agneaux nés simples, doubles et triples. Cette différence de poids à la naissance peut être expliquée par le fait qu'au niveau des naissances simples, on note un manque de compétition ce qui est tout à fait contraire au niveau des naissances doubles et triples. Par ailleurs, la supériorité du poids des agneaux nés triples à J120 s'expliquerait par la mort de deux agneaux à J60, ce qui a supprimé carrément la compétition au sein de la portée. Nos résultats corroborent ceux de (Poivey et al., 1982 ; Armbruster et al., 1991a ; Abassa et al., 1992 ; Bonfoh et al., 1996 ; Yapi-Gnaoré et al., 1997b ; Gbangboche, 2005 ) qui pensent que les agneaux nés doubles ou triples sont plus légers que les simples et aucune croissance compensatrice systématique n'est observée après sevrage. Quant au facteur âge des brebis, une différence significative $(p<0,05)$ a été observée entre les poids vifs moyens des agneaux à la naissance nés des brebis dont l'âge est compris entre 1 et 5ans. L'expérience des brebis n'a pas cependant influencé les poids de $\mathrm{J} 30$ à $\mathrm{J} 180(\mathrm{p}>0,05)$. Cette significativité des poids à la naissance pourrait être du à la taille de la portée qui est relativement élevée chez les brebis dont l'âge est situé entre 4 et 5 ans par rapport aux jeunes mères (primipares), ce qui diminuerait le poids à la naissance des agneaux nés doubles ou triples (multipares). Ces résultats sont semblable à ceux de Fall et al., 1982 ; Poivey et al., 1982 ; Filius et al., 1986 ; Abassa et al., 1992 ; London et al, 1994 ; Yapi-Gnaoré et al., 1997a. Le facteur sexe a influencé nettement $(p<0,05)$ la croissance pondérale des agneaux du début à la fin bien que cet effet semble disparaitre à J60. Nos résultats sont en accord avec ceux Adeleye et Oguntola, 1975 ; Fall et al., 1982 ; Poivey et al., 1982 ; Taïwo et al., 1982 ; Adeleye, 1984; Otesile, 1993; Gbangboche, 2005 qui stipulent que le poids à la naissance des agneaux et agnelles varient entre 1 et $2,5 \mathrm{~kg}$ et que dans les mêmes conditions d'élevage, les mâles naissent plus lourds que les femelles. Cependant, nos résultats sont contraire à ceux de Valerand et Branckaert, 1975 ; Poivey et al., 1982 qui pensent que l'effet du sexe aurait tendance à

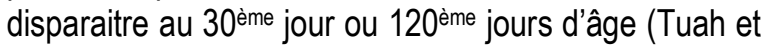
Baah, 1985). Le type de naissance a influencé de façon significative $(p<0)$ le $G M Q$ des agneaux de race 
Djallonké entre 0-30j et 90-120j. Les valeurs moyennes obtenues ont été de $126,37 \pm 51,96 ; 90,03 \pm 28,54$ et $41,67 \pm 27,28$ entre $0-30$ jours et $151,10 \pm 104,03$; $87,05 \pm 36,27$ et $23,81 \pm 82,48$ entre $90-120$ jours respectivement pour les agneaux nés uniques, doubles et triples. Ces valeurs sont similaires à celles obtenues par Vallerand et Branckaert, 1975 ; Rombaut, 1980 ; Poivey et al., 1982. Ces valeurs sont de l'ordre de 50$150 \mathrm{~g}$ le mois qui suit la naissance. Ces gains chutent par la suite en dessous de ce niveau ou restent stable dans le meilleur des cas ; entre 30 à 60 jours, ils sont supérieurs à $50 \mathrm{~g}$, entre 0 et 80 jours d'âge, de l'ordre de $70 \mathrm{~g}$ (Yapi-Gnaoré et al., 1997a), entre 0 et 90 jours, de $85 \mathrm{~g}-112 \mathrm{~g}$ pour les naissances gémellaires et 115 g-134 g pour les naissances simples (N'Gere, 1973). Quant à l'expérience des brebis, une différence significative des gains moyens quotidiens est notée entre 0-30 jours. Les meilleurs GMQ sont observés chez les agneaux dont l'âge des mères se situe entre 3 et 4 ans avec des valeurs moyennes de 113,09 $\pm 56,57$ et $123,81 \pm 29,88$ respectivement pour les brebis de 3ans et 4ans. Le GMQ le plus faible a été enregistré chez les agneaux dont l'âge de la mère était le plus élevé (5ans) suivi des agneaux issus des jeunes brebis (2ans). Ce faible GMQ chez les agneaux issus des femelles adultes (5ans) s'expliquerait par le fait que ces

\section{CONCLUSION}

À la lumière des résultats obtenus dans cette étude, on peut conclure que les performances de croissance réalisées par les agneaux de race Djallonké à la Ferme Agropastorale de Dzo-mossi sont satisfaisantes. Ces paramètres (croissance pondérale et le gain moyen

\section{REFERENCES BIBLIOGRAPHIQUES}

Abassa K.P., Pessinaba J., Adeshola-Ishola. A., 1992. Croissance pré-sevrage des agneaux Djallonké au Centre de Kolokopé (Togo). Rev. Elev. Méd. Vét. Pays Trop., 45, 49-54.

Adeleye I.O.A., 1984. Seasonal effects on lamb production under tropical conditions. Niger. J. Anim. Prod., 1984, 11, 168-174.

Adeleye L.O., Ogountona E., 1975. Effects of age and sex on liveweight and body composition of the West African Dwarf Sheep. Niger. J. Anim. Prod., 2, 264-269.

Aloulou R., 1990. Croissance pondérale de la race Barbarine dans le semi-aride Tunisien : coefficients de correction et paramètres génétiques. Mém. Cycle spécialisation, Institut national agronomique, Tunis, Tunisie, $59 \mathrm{p}$. femelles ont la probabilité de donner les triplés et la compétition au niveau du lait ralentisserait sans doute le gain de poids chez ces derniers. Quant aux faibles GMQ chez les agneaux issus des primipares, il s'expliquerait au fait que les mères elles-mêmes n'ont pas encore fini leur croissance et par conséquent elles entrent en compétition avec leurs petits d'une part, et la faible production de lait par ces primipares d'autre part. Nos résultats sont semblables à ceux de (Fall et al., 1982 ; Poivey et al., 1982 ; Filius et al., 1986 ; Abassa et al., 1992 ; London et al, 1994 ; Yapi-Gnaoré et al., 1997a) qui pensent que Les agneaux issus du premier et dans une certaine mesure du second agnelage sont généralement plus légers à la naissance et par conséquent gagnent de façon timide leurs $G M Q$ que ceux des agnelages suivants. Le facteur sexe n'a pas influencé le gain moyen quotidien à la naissance $(p>0,05)$. II a été cependant déterminant par la suite (30-180j) auquel le GMQ des males a fortement dominé sur celui des femelles $(p<0,05)$. Cette différence serait du probablement au dynamisme des males et à leurs mobilités au niveau des mamelles. Nos résultats sont similaires aux résultats rapportés par Boujenane et Mharchi, 1992 chez les agneaux de race Béni Guil au Maroc.

quotidien) ont été plus meilleurs chez les portées uniques. La croissance pondérale et le gain moyen quotidien élevés chez les naissances triples met en éclair le problème de la compétition au niveau de leur alimentation avant sevrage au sein d'une même portée.

Amoundala-Matala., 1982. Études de quelques performances zootechniques du mouton de Loukoléla en milieu villagéois. Université Marien Ngouabi, Mémoire IDR, Brazzaville.

Armbruster T., Peters K.J., Metz T., 1991a. Sheep production in the humid zone of West Africa : II. Growth performance and live weighs of sheep in improved and traditional production systems in Côte - d'Ivoire. J. Anim. Breed. Genet., 108, 210-220

Ashley S., Holden S et Bazeley P., 1999. Livestock in poverty-focused development. In Development Ltd : Chippenham, $95 \mathrm{p}$.

Batalou-Mbetani A et Bourzat D., 1989. Étude des petits ruminants dans le système traditionnel agroforestier du Mayombe, Congo. Rapport de 
recherche, 1ère partie, DGRST/CIPEA, Brazzaville, Congo, 85p.

Ben Hamouda M., 1985. Description biométrique et amélioration génétique de la croissance pondérale des ovins de race Barbarine. Thèse Doct., Université de Gand, Belgique, $166 \mathrm{p}$.

Bengaly Z., Clausen P.H., Boly H., Kwame A et Duvallet G., 1993. Comparaison de la trypanosomose expérimentale chez certaines races de petits ruminants du Burkina Faso. Rev. Elev. Méd. Vét. Pays Trop, 46, 563-570.

Bonfoh B., Traoré A., Ayewa T., 1996. Contrôle de performances, sélection des mâles au sein de la race ovine Djallonké et création d'un flockbook. In : Proceeding of the third Biennial Conference of the African Small Ruminant Research Network Workshop, 5-9 December 1994. International Livestock Research Institute (ILRI) : Nairobi, 71-78.

Boujenane. I et Mharchi. A, 1992. Estimation des paramètres génétiques et phénotypiques des performances de reproduction des brebis de race Béni Guil. Actes Inst. Agron. Vet., Vol. 12 (4), 5-13.

Centre International Pour L'élevage En Afrique (Cipea)., 1979. Le bétail trypanotolérant d'Afrique occidentale et centrale. CIPEA : Addis Abeba, 1979, 308 p.

Clement V., Poivey J.P., Faugere O., Tillard E., Lancelot R., Gueye A., Richard D et Bibe B., 1997. Étude de la variabilité des caractères de reproduction chez les petits ruminants en milieu traditionnel au Sénégal. Rev. Elev. Méd. Vét. Pays Trop, 50, 235-249.

Fall A., Gueye E., Diop M., Sandford J., Wissocq J.Y., Durquin J. Trail J.C.M., 1982. Évaluation des productivités des ovins taurins et des taurins Ndama au

Centre de Recherches zootechniques de Kolda, Sénégal. Centre International pour l'Élevage en Afrique (Cipea) : Addis-Abeba, $74 \mathrm{p}$.

Faye B et Alary V., 2001. Les enjeux des productions animales dans les pays du Sud. Prod. Anim, $14,3-13$

Faye B et Lhoste P., 1999. Le conseil en élevage en milieu tropical. In : 6e Rencontres Recherches Ruminants. Institut national de la Recherche agronomique, Institut de l'Elevage : Paris, 6367.
Filius P., Weniger J.H, Teuscher T., 1986. Investigations on the performance of Djallonké sheep. Anim. Res. Dev., 24, 85-97.

Gbangboche A.B., 2005. Performances zootechniques et paramètres génétiques des ovins Djallonké au Bénin. Thèse de doctorat, Université de Liège, 193p.

Jmal S., 1995. Paramètres génétiques des caractères de la productivité de la brebis Barbarine dans le semi-aride Tunisien. Mém. Cycle spécialisation, Institut national agronomique, Tunis, Tunisie, $84 \mathrm{p}$.

Kinga J.C., 1983. L'étude des conditions d'élevage et de quelques performances zootechniques des souches caprines des plateaux. Université Marien Ngouabi, Mémoire IDR, Brazzaville, 85p.

Lebbie S.H.B et Ramsay K., 1999. A perspective on conservation and management of small ruminant genetic resources in the sub-Saharan Africa. Small Rumin. Res, 34, 231-247.

Lhoste P., Dolle V., Rousseau J et Soltner D., 1993. Zootechnie des régions chaudes: les systèmes d'élevage, Ministère de la coopération, Paris, France, pp. 26-27.

London J.C., Weniger J.H., Schwartz H.J.,1994. Investigation into traditionnaly managed Djallonkésheep production in humid and subhumid zones of Asante, Ghana. II. Reproductive events and prolificacy. J. Anim. Breed. Genet., 111, 432-450.

Mawuena K., 1986. Trypanosomose des moutons et des chèvres de race naine Djallonké des régions sud guinéennes du Togo. Rev. Elev. Méd. Vét. Pays Trop, 39, 307- 315.

Mawuena K., 1987. Haut degré de tolérance à la trypanosomose des moutons et des chèvres de race Naine Djallonké des régions sudguinéennes du Togo : comparaison avec les bovins trypanotolérants. Rev. Elev. Méd. Vét. Pays Trop, 40, 55-58.

Mfoukou-Ntsakala A., 2010. Contribution à l'étude de l'élevage urbain de petits ruminants et leur affouragement avec de l'ensilage au Congo. Thèse de doctorat, Université Marien Ngouabi, Brazzaville, 184p.

N'gere L. O., 1973. Size and growth rate of the west African dwarf sheep and new breed, the Nungua black head of Ghana. Ghana J. Agric. Sci., 6, 113-117. 
Otesile E.B., 1993. Studies on West African Dwarf sheep: the influence of age of ewe and parturition interval on neonatal lamb mortality rate. Bull. Anim. Prod. Afr., 41, 251-255.

Poivey J.P., Landais E., Berger Y., 1982. Étude et amélioration génétique de la croissance des Djallonké. Résultats obtenus au Centre de Recherches Zootechniques de Bouaké (Côted'Ivoire). Rev. Elev. Méd. Vét. Pays Trop., 35, 421-433.

Rombaut D., 1980. Comportement du mouton Djallonké en élevage rationnel. Rev. Elev. Méd. Vét. Pays Trop., 33, 427-439.

Savou D.D., 1982. Contribution au développement de l'élevage ovin et caprin en milieu paysan : Cas des pays du Niari. Mémoire IDR, Université Marien Ngouabi, Brazzaville.

Taiwo B.B.A., Ngere L.O., Adeleye I.Oa., 1982. Comparative growth performance of Nigerian dwarf sheep and its crosses with Permer, Uda and Yankassa. Word Rev. Anim. Prod., 18, 5763.

Tuah A.K., Baah. J., 1985. Reproduction performance, preweaning growth rate and pre-weaning lamb mortality of Djallonke sheep in Ghana. Trop. Anim. Health Prod., 17, 107-113.

Vallerand F., Branckaert R., 1975. La race ovine Djallonké au Cameroun. Potentialités zootechniques, conditions d'élevage, avenir. Revue. Elev. Méd. Vét. Pays trop., 1975, 28, 523-545.

Wilson R.T., 1983. Studies on the livestock of Southern Darfur, Sudan. VIII A comparison of productivity indices for goats and sheep Trop. Anim. Health Prod, 15, 63-68.

Wilson R.T.,1989. Productive performance of African indigenious small ruminants under various management systems. Rev. Anim. Reprod. Sci, 20, 265-286.

Yapi-Gnaore C.V., Oya A., Rege J.E.O., Dagnogo B., 1997a. Analysis of an open nucleus-breeding programme for Djallonke sheep in the Ivory Coast. 1. Examination of non-genetics factors. Anim. Sci, 64, 291-300.

Yapi-Gnaore C.V., Rege J.E., Oya A., Alemayelu N., 1997b. Analysis of an open nucleus-breeding programme for Djallonke sheep in the Ivory Coast. 2. Response to selection on body weights. Anim. Sci, 64, 301- 307. 